OPEN ACCESS

Edited by:

Aaron Tan,

University College London, UK

Reviewed by:

Yong $L i$,

University of Texas Health Science Center at Houston, USA

Christopher D. Porada,

Wake Forest School of Medicine, USA

*Correspondence:

António J. Salgado

asalgado@ecsaude.uminho.pt

Specialty section:

This article was submitted to

Integrative and Regenerative

Pharmacology,

a section of the journal

Frontiers in Pharmacology

Received: 29 April 2016

Accepted: 15 July 2016

Published: 03 August 2016

Citation:

Marote A, Teixeira FG,

Mendes-Pinheiro $B$ and Salgado AJ (2016) MSCs-Derived Exosomes: Cell-Secreted Nanovesicles with

Regenerative Potential.

Front. Pharmacol. 7:231

doi: 10.3389/fphar.2016.00231

\section{MSCs-Derived Exosomes: Cell-Secreted Nanovesicles with Regenerative Potential}

\author{
Ana Marote 1,2, Fábio G. Teixeira ${ }^{1,2}$, Bárbara Mendes-Pinheiro ${ }^{1,2}$ and \\ António J. Salgado1,2* \\ 1 Life and Health Sciences Research Institute (ICVS), School of Health Sciences, University of Minho, Braga, Portugal, \\ 2 ICVS/3B's, PT Government Associate Laboratory, Braga/Guimarães, Portugal
}

Exosomes are membrane-enclosed nanovesicles (30-150 nm) that shuttle active cargoes between different cells. These tiny extracellular vesicles have been recently isolated from mesenchymal stem cells (MSCs) conditioned medium, a population of multipotent cells identified in several adult tissues. MSCs paracrine activity has been already shown to be the key mediator of their elicited regenerative effects. On the other hand, the individual contribution of MSCs-derived exosomes for these effects is only now being unraveled. The administration of MSCs-derived exosomes has been demonstrated to restore tissue function in multiple diseases/injury models and to induce beneficial in vitro effects, mainly mediated by exosomal-enclosed miRNAs. Additionally, the source and the culture conditions of MSCs have been shown to influence the regenerative responses induced by exosomes. Therefore, these studies reveal that MSCs-derived exosomes hold a great potential for cell-free therapies that are safer and easier to manipulate than cell-based products. Nevertheless, this is an emerging research field and hence, further studies are required to understand the full dimension of this complex intercellular communication system and how it can be optimized to take full advantage of its therapeutic effects. In this mini-review, we summarize the most significant new advances in the regenerative properties of MSCs-derived exosomes and discuss the molecular mechanisms underlying these effects.

Keywords: exosomes, mesenchymal stem cells, secretome, regeneration, cell-free therapy

\section{INTRODUCTION}

Exosomes are the tiniest extracellular vesicles (EVs) involved in a complex intercellular communication system (Lamichhane et al., 2015). EVs are categorized as exosomes due to their endocytic origin, in contrast to microvesicles that are formed from budding of the plasma membrane and apoptotic bodies derived from fragments of dying cells (Merino et al., 2016). Along with other EVs, exosomes are released by several cell types, carrying active signals that are able to influence the activity of recipient cells. Since its discovery, this extracellular communication system has been hijacked in several ways. Firstly, exosome surface markers and molecular cargoes have been worthily challenged as potential diagnostic biomarkers (Muller, 2012; Dorayappan et al., 2016; Escudero et al., 2016). Secondly, due to their low immunogenicity, long half-life in circulation and ability to cross the brain-blood barrier (BBB), exosomes have also been explored as a nanodelivery system of therapeutic signals, such as small interfering RNAs (Sun et al., 2010; 
Alvarez-Erviti et al., 2011; Kalani et al., 2014). Finally, and surprisingly, unmodified exosomes secreted by stem or progenitor cells have also shown beneficial effects and have been put forward as mediators of the regenerative responses elicited, for instance, by mesenchymal stem cells (MSCs; Lai et al., 2010; Xin et al., 2012). Indeed, the effects of these cells on tissue regeneration and repair have been mainly attributed to their secreted factors (known as secretome) rather than its transdifferentiation capacity (Teixeira et al., 2013). Having this in mind, under the scope of the present review we will address the recent evidences of the regenerative potential of MSCs-derived exosomes into different pathological conditions.

\section{EXOSOMES AS CELL-SECRETED EVs}

Exosomes and microvesicles are the most thoroughly studied classes of EVs. Both are membrane-enclosed vesicles, surrounded by a phospholipid layer and packed with cell-type specific combinations of proteins, lipids, coding, and non-coding RNAs (Lener et al., 2015). Once released into the extracellular milieu, EVs can be taken up by target cells of the microenvironment or carried to distant sites through biological fluids, from which they have already been isolated, including urine (Royo et al., 2016), breast milk (Zonneveld et al., 2014), blood (Baranyai et al., 2015), and cerebrospinal fluid (Street et al., 2012).

In spite of their relative availability, the complexity of the extracellular environment and the existence of extracellular RNA in other non-EV carriers, such as protein complexes and lipoproteins, has encouraged the International Society for Extracellular Vesicles (ISEV; Lötvall et al., 2014) to establish minimal requirements for describing EVs, namely: (i) isolation from extracellular body fluids or conditioned cell culture medium, with minimal cell disruption; (ii) quantification of at least one protein from three different categories (transmembrane or lipid bound extracellular proteins, cytosolic proteins and intracellular proteins) in the EV preparation; (iii) characterization of single vesicles within the EV preparation using at least two different technologies, electron microscopy or atomic force microscopy (AFM) for imaging and nanoparticletracking analysis, dynamic light scattering, or resistive pulse sensing for size distribution measurements of EVs (Lötvall et al., 2014). EVs are further classified as exosomes or microvesicles based on their intracellular biogenesis pathway. Microvesicles are originated at the cell surface and released by direct outward budding of the plasma membrane, whereas exosomes are formed within multivesicular bodies (MVBs) at the endolysosomal pathway and secreted upon fusion of MVBs with the plasma membrane (Figure 1A; Kalani et al., 2014).

Exosomes comprise a homogenous population of nano-sized vesicles, as their diameter ranges from 30 to $150 \mathrm{~nm}$ (Lener et al., 2015; Willms et al., 2016). Additionally, exosomes are also a better characterized population of EVs. Exosomal proteins, RNAs and lipids described in published and unpublished studies have been cataloged in ExoCarta, a database that aims to identify specific molecular signatures of tissue/cell-type derived exosomes (Mathivanan et al., 2012). ALIX and TSG10 have been recognized as common protein markers for exosome identification, due to their participation in the endosomal sorting complex required for transport (ESCRT), as well as membrane proteins of the tetraspanin family, such as CD9, CD63, CD81, CD82 (Figure 1B; Wubbolts et al., 2003; Hurley and Odorizzi, 2012).

Conventional methods for isolation of exosomes include ultracentrifugation, density gradient separation, chromatography and immunoaffinity capture (IAC) techniques as well as commercial kits, such as polymer-based precipitation. Despite being all capable to isolate vesicles with exosomal diameter and markers, some authors have suggested IAC as the most effective method to isolate exosomes, based on the number of exosomerelated proteins identified on the purified fraction (Tauro et al., 2012; Greening et al., 2015). However, there is an ongoing debate on the ability of these methods to fully separate exosomes from protein aggregates and other membranous vesicles. The distinction between microvesicles and exosomes, for instance, is challenging as a result of the heterogeneity of microvesicles diameter (50-1000 nm), as well as small amount of protein markers to clearly categorize EVs produced via budding from the cell membrane or produced via endosomal compartment. Therefore, the ISEV (Lötvall et al., 2014) has recommended cautious interpretation of some exosomal-mediated responses described so far. In fact, some studies focused on the evaluation of the individual contribution of exosomes and microvesicles, report unique effects of exosomes- and microvesicles- enriched fractions that were obtained through differential centrifugation steps (Jarmalaviciute et al., 2015; Lopez-Verrilli et al., 2016). Even within the same population of exosomes, Willms et al. (2016) have defined two distinct subpopulations of exosomes based on distinct particles sizes that enclose different protein and RNA compositions and, therefore, yield different effects on recipient cells. Taken together, these studies show that the improvement of current isolation methods and a better understanding of exosomal biology are of key importance to clearly determine the therapeutic effects of exosomes.

\section{MSCs and MSCs-DERIVED EXOSOMES}

The regenerative potential of MSCs and particularly of their secretome has been extensively reviewed (Teixeira et al., 2013; Salgado et al., 2015; Konala et al., 2016). MSCs are a population of adult multipotent cells with the ability to self-renew and differentiate into mesenchymal lineages, namely osteoblasts, chondrocytes, and adipocytes (Pittenger et al., 1999). Even though originally isolated from bone marrow (BM-MSCs; Friedenstein et al., 1976), MSCs have been successfully identified and isolated from other adult tissues, such as adipose tissue (ASCs), dental pulp, placenta, amniotic fluid, umbilical cord blood, umbilical cord Wharton's jelly (WJ-MSCs) and its perivascular region (HUCPVCs), and even the brain (Teixeira et al., 2013). MSCs differentiation potential has been explored in cell replacement strategies aiming to restore compromised adult mesenchymal tissues, although some authors have also reported its differentiation into ectodermal lineages (Donega et al., 2014; Takeda and Xu, 2015; Bagher et al., 2016). 
A

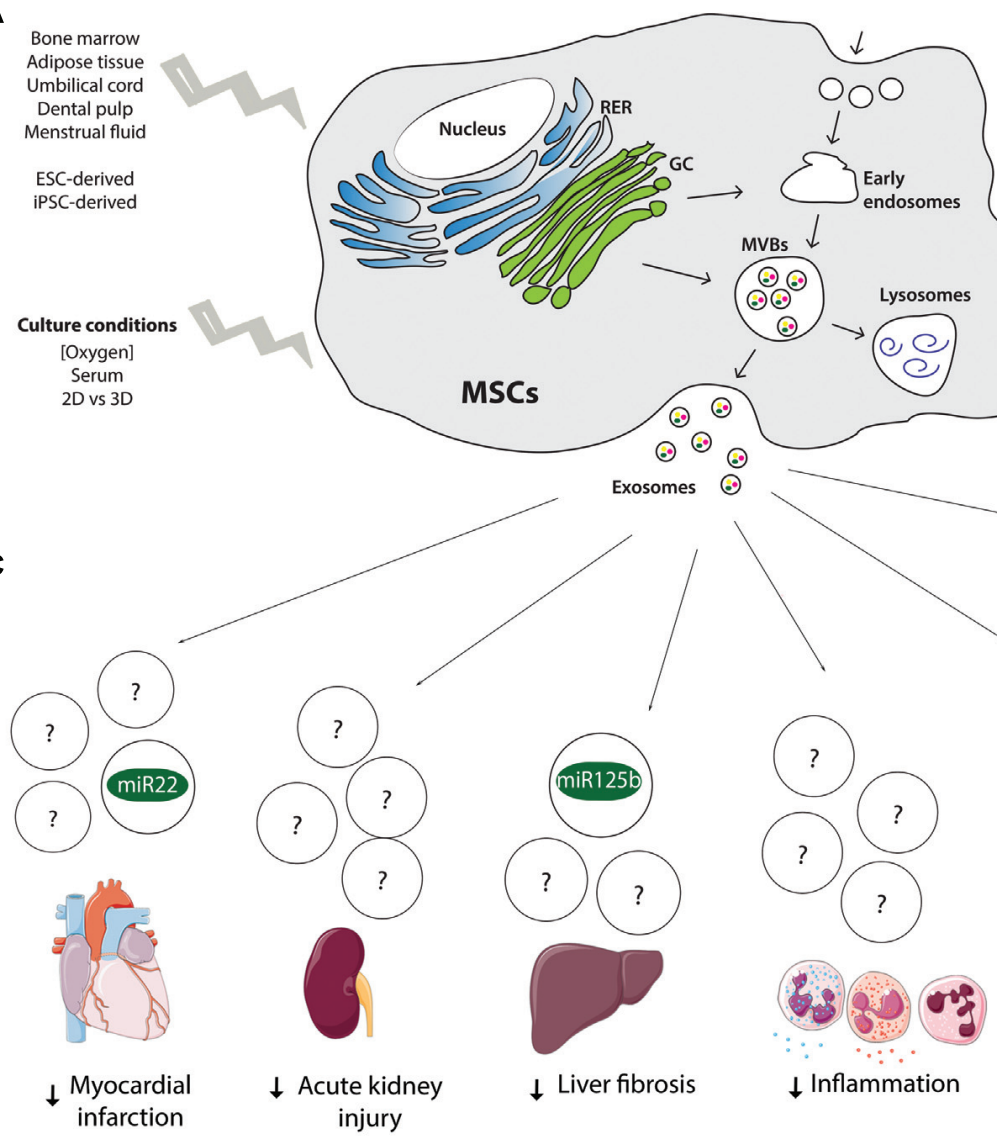

B

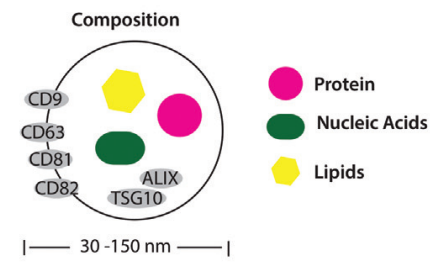

C 
BM-MSCs and ASCs exosomes, recently performed by Baglio et al. (2015), revealed that, along with miRNA, MSCs exosomes are highly enriched in transfer RNA (tRNA) species that can function as miRNAs. Furthermore, while cellular small RNA expression profile is not donor- or tissue-specific, exosomal small RNA content seems to be greatly influenced by cell differentiation status, i.e., donor variability, and cell type (Baglio et al., 2015). The authors suggest that exosomal-enclosed small RNAs released by MSCs may distinctly control the microenvironment in their resident niches through a balance between proliferation and differentiation (Baglio et al., 2015). This study shed light on possible regulatory mechanisms of MSCs paracrine activity responsible for tissue-specific MSCs CM regenerative properties that were previously described by our laboratory (Ribeiro et al., 2012). Recently, tissue-specific responses were also described for exosomes isolated from different sources. For instance, ASCs-derived exosomes seem to be more effective in degrading $\mathrm{A} \beta$ in an in vitro model of Alzheimer's disease (AD), when compared to BM-MSCs-derived exosomes (Katsuda et al., 2013), whereas neurite outgrowth response appears to be enhanced by exosomes released by menstrual fluid derived MSCs, when compared to umbilical cord, chorion and bone marrow (LopezVerrilli et al., 2016). These studies unveil the need to further distinguish the functional properties of exosomes released by MSCs isolated from different adult tissues to better understand their individual regenerative potential. The influence of gender on MSCs exosomal secretion profile should be also taken into account in future studies.

\section{MSCS-DERIVED EXOSOMES APPLIED TO TISSUE REGENERATION}

Since the report of MSCs-derived exosomes, a growing amount of studies have explored their regenerative potential using different in vitro and in vivo models (Figure 1C). In the present report we will focus on those that, in our opinion, are the most relevant.

\section{Cardiovascular System}

MSCs-derived exosomes were firstly suggested as cardioprotective agents by Kleijn's group (Lai et al., 2010). Subsequently, other studies have addressed the proangiogenic effects of MSCs exosomes as a mechanism of tissue repair upon ischemia. Bian et al. (2014) firstly showed that MSCs secrete a large quantity of exosomes under hypoxia and serum deprivation. In a similar ischemic conditioning, MSCs were shown to secrete exosomes enriched with miR-22, which led to the reduction of apoptosis and cardiac fibrosis through a direct targeting of methyl CpG binding protein 2 (Mecp2; Feng et al., 2014). In line with this, a recent analysis of the proteomic profile of MSCs-derived exosomes cultured under these conditions revealed a robust profile of angiogenic signaling proteins, including $\mathrm{NFkB}$ interacting proteins, which mediate tubule formation upon low to medium doses $(1-10 \mu \mathrm{g} / \mathrm{mL}$ ) treatment of MSCs-derived exosomes (Anderson et al., 2016).

Importantly, several authors have demonstrated that internalization of MSCs-derived exosomes by endothelial cells stimulate proliferation, migration and tube formation (in vitro) and promote angiogenesis when injected intramyocardially in acute myocardial infarction rat models (Bian et al., 2014; Teng et al., 2015). Hu et al. (2015) have also evidenced that intramuscular injection of exosomes secreted by human induced pluripotent stem cells-derived MSCs (iPSCs-derived MSCs) promote endothelial cell migration, proliferation, and tube formation through the activation of angiogenesis-related molecule expression (VEGF, TGFB1, and Angiogenin), leading to an increase of microvessel density and blood perfusion in a mouse hind-limb ischemic model.

In a follow-up study, Arslan et al. (2013) have shown that a single intravenous administration of exosomes was effective in enhancing cardiac function and geometry after myocardial infarction due to bioenergetics reestablishment (increased ATP production), oxidative stress reduction and pro-survival signaling activation (enhanced PI3K/Akt signaling). A similar outcome was presented by Wang et al. (2015) in polymicrobial sepsis.

Asahara's group (Zhang et al., 2016b) have used the proliferative and angiogenic potential of MSCs exosomes to condition cardiac stem cells (CSC) in vitro, which when transplanted into a rat model of myocardial infarction were able to preserve cardiac function.

\section{Kidney}

Acute kidney injury has also been effectively treated with MSCs exosomes. In vitro, treated renal tubular epithelial cells exhibited increased activation of extracellular-signal-regulated kinase (ERK)1/2 pathway and thus increased cell proliferation (Zhou et al., 2013). In vivo, after direct injection into injured kidneys (cisplatin-induced acute kidney injury rat model), hucMSCs-Ex were shown to be incorporated into the tubular epithelial cells and to promote functional and morphologic recovery by reducing oxidative stress and suppressing apoptosis (Zhou et al., 2013). Recently, Jiang et al. (2016), characterized urine-derived stem cells as multipotent MSCs (USCs) and a source of exosomes, and demonstrated that when intravenously injected they were able to reduce kidney deficits in type I diabetic rats.

\section{Liver}

HucMSCs-Ex have also been explored as a treatment to fibrotic liver disease, which can be induced by viral hepatitis, alcohol, drugs, metabolic diseases, and autoimmune attack of hepatic cells (Li et al., 2013). Li et al. (2013), have demonstrated that after liver fibrosis [induced by carbon tetrachloride $(\mathrm{CCl} 4)$ ], the delivery of exosomes into the liver reduced fibrosis through the inactivation of the transforming growth factor (TGF) $\beta$ 1/Smad signaling pathway, as well as through the inhibition of epithelialto-mesenchymal transition of hepatocytes. Using the same mouse model, Hyun et al. (2015) demonstrated that chorionic platederived MSCs retained miR-125b in exosomes which suppressed the Hedgehog activation and consequently liver fibrosis. In addition to this, the hepatic regenerative potential of hESCderived MSCs exosomes was also evidenced using two in vitro models of liver injury [acetaminophen (APAP)- and (2) hydrogen 
peroxide $\left(\mathrm{H}_{2} \mathrm{O}_{2}\right)$ ], in which treatment resulted in increased antiapoptotic and proliferative responses (Tan et al., 2014).

\section{Immune System}

In accordance to well-known MSCs immunomodulatory effects, MSCs exosomes have also been described as immunological active agents as they induce high levels of anti-inflammatory cytokines, in contrast to decreased levels of pro-inflammatory molecules, providing a rationale for the use of exosomes for the treatment of immune disorders (Zhang et al., 2014). In addition, MSCs exosomes can also inhibit macrophage activation by suppressing Toll-like receptor signaling, providing a physiologically relevant context for the innate immunomodulatory activity of MSCs (Phinney et al., 2015). In a mouse model of hypoxic pulmonary hypertension, Lee et al. (2012) demonstrated that the intravenous delivery of exosomes derived from MSCs CM suppress hypoxic inflammation by inhibition of pro-proliferative pathways, such as STAT3 phosphorylation.

\section{Musculoskeletal System}

In the context of bone regeneration, Narayanan et al. (2016) demonstrated the potential of exosomes released by MSCs cultured under osteogenic conditions. In a 2D and in a 3D (type I collagen hydrogels) culture these exosomes triggered osteogenic differentiation of undifferentiated MSCs. In vivo, when implanted subcutaneously in a grade collagen membrane, "osteogenic" exosomes promoted calcium deposition and calcium phosphate nucleation. In addition, Nakamura et al. (2015) have also suggested that MSCs-derived exosomes are able to promote skeletal muscle regeneration by enhancing myogenesis and angiogenesis and have considered exosome-enclosed miR-494 an important mediator of this response.

\section{Nervous System}

MSCs-secretome has shown therapeutic benefits for the treatment of neurological and neurodegenerative diseases. Accordingly, Xin et al. (2012, 2013b) have demonstrated that miR-133b, increased in MSCs exposed to ischemic cerebral extracts, is transferred to neurons and astrocytes via exosomes and promotes neurite outgrowth and functional recovery in a middle cerebral artery occlusion model. In another study, these authors (Xin et al., 2013a) demonstrated that after stroke, the intravenous administration of cell-free MSCs-generated exosomes also enhances neurite remodeling, neurogenesis and angiogenesis and therefore, improves functional recovery. Similar encouraging outcomes were obtained in a traumatic brain injury model, in which intravenous administration of MSCsgenerated exosomes enhanced angiogenesis and neurogenesis, reduced inflammation and improved spatial learning and sensimotor function (Zhang et al., 2015; Kim et al., 2016).

Katsuda et al. (2013) have provided an important role for ASCs-derived exosomes in the context of $\mathrm{AD}$, as these express high levels of Neprilysin (NEP), the most important $\mathrm{A} \beta$-degrading enzyme in the brain. NEP-loaded exosomes were shown to be efficiently transferred to neuroblastoma cells and led to the decrease of both extracellular and intracellular $A \beta$ levels. In another in vitro neurodegeneration model, exosomes released by dental pulp-derived MSCs on a 3D culture, rescued dopaminergic neurons from 6-OHDA induced apoptosis, thereby providing a possible treatment of Parkinson's disease (Jarmalaviciute et al., 2015).

Other studies have focused on non-diseased in vitro models to demonstrate MSCs-derived exosomes potential for neuronal differentiation. For instance, Lee et al. (2014) reported that BMMSCs deliver miR-124 and miR-145 to human neural progenitor cells (NPCs) and astrocytes via contact independent and exosome-dependent processes, which alter gene expression in the recipient neural cells. Indeed, exosomal-mediated axonal growth seems to depend on argonaut 2 protein, a miRNA machinery protein, whereas internalization of BM-MSCs exosomes in cell bodies and axons has been suggested to occur via SNARE complex (Zhang et al., 2016a). Lopez-Verrilli et al. (2016) have recently unraveled the potential of menstrual MSCs (MenSCs) exosomal-enriched fraction as therapeutic approach in neurodegenerative pathologies. These authors demonstrated that MenSC exosomes induced neurite growth in cortical neurons and had a similar effect to BM-SC exosomes on neurite outgrowth of dorsal root ganglia neurons.

\section{CONCLUSION AND FUTURE PERSPECTIVES}

MSCs-derived exosomes have emerged, in the last six years, as an attractive and safe approach for regenerative medicine applications. Of note, the intravenous administration of these nanovesicles has not elicited adverse effects. In addition, MSCs-derived exosomes regenerative effects reinforce the paradigm of secretome-based paracrine action of MSCs and support future perspectives for the development of cell-free therapies that avoid the safety concerns associated to stem cell transplantation and are less technically difficult to prepare and deliver. Nevertheless, this is a still developing research area that needs to be optimized to take full advantage of MSCs-derived exosomes regenerative potential. For instance, the choice of MSCs source and their culture conditions must be explored, as these have been shown to impact the functional properties of the exosomes.

\section{AUTHOR CONTRIBUTIONS}

AM drafted the manuscript. FT helped to draft the manuscript and revised it critically. BM-P collected all scientific literature for review. AS conceived the analysis, participated in its design and coordination, helped to draft the manuscript and gave the final approval of the version to be published. All authors read and approved the final manuscript.

\section{ACKNOWLEDGMENTS}

Prémios Santa Casa Neurociências - Prize Melo e Castro for Spinal Cord Injury Research; Portuguese Foundation for Science 
and Technology (Doctoral fellowship - PDE/BDE/113598/2015 to AM. IF Development Grant to AS). This article is a result of the project (NORTE-01-0145-FEDER-000013), supported by Norte Portugal Regional Operational Programme (NORTE 2020), under the PORTUGAL 2020 Partnership Agreement, through the European Regional Development Fund (ERDF) Cofinanciado pelo Programa Operacional Regional do Norte (ON.2 SR\&TD Integrated Program - NORTE-070124-FEDER-000021), ao abrigo do Quadro de Referência

\section{REFERENCES}

Alvarez-Erviti, L., Seow, Y., Yin, H., Betts, C., Lakhal, S., and Wood, M. J. A. (2011). Delivery of siRNA to the mouse brain by systemic injection of targeted exosomes. Nat. Biotech. 29, 341-345. doi: 10.1038/nbt.1807

Anderson, J. D., Johansson, H. J., Graham, C. S., Vesterlund, M., Pham, M. T., Bramlett, C. S., et al. (2016). Comprehensive proteomic analysis of mesenchymal stem cell exosomes reveals modulation of angiogenesis via nuclear factor-kappab signaling. Stem Cells 34, 601-613. doi: 10.1002/stem. 2298

Arslan, F., Lai, R. C., Smeets, M. B., Akeroyd, L., Choo, A., Aguor, E. N. E., et al. (2013). Mesenchymal stem cell-derived exosomes increase ATP levels, decrease oxidative stress and activate PI3K/Akt pathway to enhance myocardial viability and prevent adverse remodeling after myocardial ischemia/reperfusion injury. Stem Cell Res. 10, 301-312. doi: 10.1016/j.scr.2013.01.002

Bagher, Z., Azami, M., Ebrahimi-Barough, S., Mirzadeh, H., Solouk, A., Soleimani, M., et al. (2016). Differentiation of wharton's jelly-derived mesenchymal stem cells into motor neuron-like cells on three-dimensional collagen-grafted nanofibers. Mol. Neurobiol. 53, 2397-2408. doi: 10.1007/s12035-015-9199-x

Baglio, S. R., Rooijers, K., Koppers-Lalic, D., Verweij, F. J., Perez Lanzon, M., Zini, N., et al. (2015). Human bone marrow- and adipose-mesenchymal stem cells secrete exosomes enriched in distinctive miRNA and tRNA species. Stem Cell Res. Ther. 6, 127. doi: 10.1186/s13287-015-0116-z

Baranyai, T., Herczeg, K., Onodi, Z., Voszka, I., Modos, K., Marton, N., et al. (2015). Isolation of exosomes from blood plasma: qualitative and quantitative comparison of ultracentrifugation and size exclusion chromatography methods. PLoS ONE 10:e0145686. doi: 10.1371/journal.pone.0145686

Bian, S., Zhang, L., Duan, L., Wang, X., Min, Y., and Yu, H. (2014). Extracellular vesicles derived from human bone marrow mesenchymal stem cells promote angiogenesis in a rat myocardial infarction model. J. Mol. Med. (Berl.) 92, 387-397. doi: 10.1007/s00109-013-1110-5

Caplan, A. I., and Dennis, J. E. (2006). Mesenchymal stem cells as trophic mediators. J. Cell. Biochem. 98, 1076-1084. doi: 10.1002/jcb.20886

Donega, V., Nijboer, C. H., Braccioli, L., Slaper-Cortenbach, I., Kavelaars, A., van Bel, F., et al. (2014). Intranasal administration of human MSC for ischemic brain injury in the mouse: in vitro and in vivo neuroregenerative functions. PLoS ONE 9:e112339. doi: 10.1371/journal.pone.0112339

Dorayappan, K. D., Wallbillich, J. J., Cohn, D. E., and Selvendiran, K. (2016). The biological significance and clinical applications of exosomes in ovarian cancer. Gynecol. Oncol. 142, 199-205. doi: 10.1016/j.ygyno.2016.03.036

Escudero, C. A., Herlitz, K., Troncoso, F., Acurio, J., Aguayo, C., Roberts, J. M., et al. (2016). Role of extracellular vesicles and microRNAs on dysfunctional angiogenesis during preeclamptic pregnancies. Front. Physiol. 7:98. doi: 10.3389/fphys.2016.00098

Feng, Y., Huang, W., Wani, M., Yu, X., and Ashraf, M. (2014). Ischemic preconditioning potentiates the protective effect of stem cells through secretion of exosomes by targeting Mecp2 via miR-22. PLoS ONE 9:e88685. doi: 10.1371/journal.pone.0088685

Friedenstein, A. J., Gorskaja, J. F., and Kulagina, N. N. (1976). Fibroblast precursors in normal and irradiated mouse hematopoietic organs. Exp. Hematol. 4, 267274.

Greening, D. W., Xu, R., Ji, H., Tauro, B. J., and Simpson, R. J. (2015). "A protocol for exosome isolation and characterization: evaluation of ultracentrifugation, density-gradient separation, and immunoaffinity capture
Estratégico Nacional (QREN), através do Fundo Europeu de Desenvolvimento Regional (FEDER); Projeto Estratégico - LA 26 - 2011-2012 and Projeto Estratégico - LA 26 - 2013-2014 cofinanciado por fundos nacionais, através da Fundação para a Ciência e a Tecnologia (PEst-C/SAU/LA0026/2011; PEst-C/SAU/LA0026/2013), e pelo Fundo Europeu de Desenvolvimento Regional (FEDER), através do COMPETE (FCOMP-01-0124-FEDER-022724; FCOMP-01-0124-FEDER037298).

methods," in Proteomic Profiling: Methods and Protocols, ed. A. Posch (New York, NY: Springer New York), 179-209.

Ha, M., and Kim, V. N. (2014). Regulation of microRNA biogenesis. Nat. Rev. Mol. Cell Biol. 15, 509-524. doi: 10.1038/nrm3838

Hu, G. W., Li, Q., Niu, X., Hu, B., Liu, J., Zhou, S. M., et al. (2015). Exosomes secreted by human-induced pluripotent stem cell-derived mesenchymal stem cells attenuate limb ischemia by promoting angiogenesis in mice. Stem Cell Res. Ther. 6, 10. doi: $10.1186 /$ scrt546

Hurley, J. H., and Odorizzi, G. (2012). Get on the exosome bus with ALIX. Nat. Cell Biol. 14, 654-655. doi: 10.1038/ncb2530

Hyun, J., Wang, S., Kim, J., Kim, G. J., and Jung, Y. (2015). MicroRNA125bmediated Hedgehog signaling influences liver regeneration by chorionic plate-derived mesenchymal stem cells. Sci. Rep. 5, 14135. doi: 10.1038/srep 14135

Jarmalaviciute, A., Tunaitis, V., Pivoraite, U., Venalis, A., and Pivoriunas, A. (2015). Exosomes from dental pulp stem cells rescue human dopaminergic neurons from 6-hydroxy-dopamine-induced apoptosis. Cytotherapy 17, 932939. doi: 10.1016/j.jcyt.2014.07.013

Jiang, Z. Z., Liu, Y. M., Niu, X., Yin, J. Y., Hu, B., Guo, S. C., et al. (2016). Exosomes secreted by human urine-derived stem cells could prevent kidney complications from type I diabetes in rats. Stem Cell Res. Ther. 7, 24. doi: 10.1186/s13287-016-0287-2

Kalani, A., Tyagi, A., and Tyagi, N. (2014). Exosomes: mediators of neurodegeneration, neuroprotection and therapeutics. Mol. Neurobiol. 49, 590-600. doi: 10.1007/s12035-013-8544-1

Katsuda, T., Tsuchiya, R., Kosaka, N., Yoshioka, Y., Takagaki, K., Oki, K., et al. (2013). Human adipose tissue-derived mesenchymal stem cells secrete functional neprilysin-bound exosomes. Sci. Rep. 3, 1197. doi: 10.1038/srep01197

Kim, D.-K., Nishida, H., An, S. Y., Shetty, A. K., Bartosh, T. J., and Prockop, D. J. (2016). Chromatographically isolated CD63+CD81+ extracellular vesicles from mesenchymal stromal cells rescue cognitive impairments after TBI. Proc. Natl. Acad. Sci. U.S.A. 113, 170-175. doi: 10.1073/pnas.1522297113

Konala, V. B., Mamidi, M. K., Bhonde, R., Das, A. K., Pochampally, R., and Pal, R. (2016). The current landscape of the mesenchymal stromal cell secretome: a new paradigm for cell-free regeneration. Cytotherapy 18, 13-24. doi: 10.1016/j.jcyt.2015.10.008

Lai, R. C., Arslan, F., Lee, M. M., Sze, N. S., Choo, A., Chen, T. S., et al. (2010). Exosome secreted by MSC reduces myocardial ischemia/reperfusion injury. Stem Cell Res. 4, 214-222. doi: 10.1016/j.scr.2009.12.003

Lamichhane, T. N., Sokic, S., Schardt, J. S., Raiker, R. S., Lin, J. W., and Jay, S. M. (2015). Emerging roles for extracellular vesicles in tissue engineering and regenerative medicine. Tissue Eng. Part B Rev. 21, 45-54. doi: 10.1089/ten.TEB.2014.0300

Lee, C., Mitsialis, S. A., Aslam, M., Vitali, S. H., Vergadi, E., Konstantinou, G., et al. (2012). Exosomes mediate the cytoprotective action of mesenchymal stromal cells on hypoxia-induced pulmonary hypertension. Circulation 126, 2601-2611. doi: 10.1161/CIRCULATIONAHA.112.114173

Lee, H. K., Finniss, S., Cazacu, S., Xiang, C., and Brodie, C. (2014). Mesenchymal stem cells deliver exogenous miRNAs to neural cells and induce their differentiation and glutamate transporter expression. Stem Cells Dev. 23, 28512861. doi: $10.1089 /$ scd.2014.0146

Lener, T., Gioma, M., Aigner, L., Börger, V., Buzas, E., Camussi, G., et al. (2015). Applying extracellular vesicles based therapeutics in clinical trials - an ISEV position paper. J. Extracell. Vesicles 4:30087. doi: 10.3402/jev.v4.30087 
Li, T., Yan, Y., Wang, B., Qian, H., Zhang, X., Shen, L., et al. (2013). Exosomes derived from human umbilical cord mesenchymal stem cells alleviate liver fibrosis. Stem Cells Dev. 22, 845-854. doi: 10.1089/scd.2012. 0395

Lopez-Verrilli, M. A., Caviedes, A., Cabrera, A., Sandoval, S., Wyneken, U., and Khoury, M. (2016). Mesenchymal stem cell-derived exosomes from different sources selectively promote neuritic outgrowth. Neuroscience 320, 129-139. doi: 10.1016/j.neuroscience.2016.01.061

Lötvall, J., Hill, A. F., Hochberg, F., Buzás, E. I., Di Vizio, D., Gardiner, C., et al. (2014). Minimal experimental requirements for definition of extracellular vesicles and their functions: a position statement from the International Society for Extracellular Vesicles. J. Extracell. Vesicles 3:26913. doi: 10.3402/jev.v3.26913

Mathivanan, S., Fahner, C. J., Reid, G. E., and Simpson, R. J. (2012). ExoCarta 2012: database of exosomal proteins, RNA and lipids. Nucleic Acids Res. 40, D1241-D1244. doi: 10.1093/nar/gkr828

Merino, C., Zuñiga, F. A., Escudero, C. A., Ormazabanl, V., Reyes, C., Salomon, C., et al. (2016). Mesenchymal stem cell-derived extracellular vesicles promote angiogenesis: new alternatives for application to human health. Front. Physiol. 7:24. doi: 10.3389/fphys.2016.00024

Muller, G. (2012). Microvesicles/exosomes as potential novel biomarkers of metabolic diseases. Diabetes Metab. Syndr. Obes. 5, 247-282. doi: 10.2147/DMSO.S32923

Nakamura, Y., Miyaki, S., Ishitobi, H., Matsuyama, S., Nakasa, T., Kamei, N., et al. (2015). Mesenchymal-stem-cell-derived exosomes accelerate skeletal muscle regeneration. FEBS Lett. 589, 1257-1265. doi: 10.1016/j.febslet.2015. 03.031

Narayanan, R., Huang, C.-C., and Ravindran, S. (2016). Hijacking the cellular mail: exosome mediated differentiation of mesenchymal stem cells. Stem Cells Int. 2016:3808674. doi: 10.1155/2016/3808674

Phinney, D. G., Di Giuseppe, M., Njah, J., Sala, E., Shiva, S., St Croix, C. M., et al. (2015). Mesenchymal stem cells use extracellular vesicles to outsource mitophagy and shuttle microRNAs. Nat. Commun. 6, 8472. doi: $10.1038 /$ ncomms 9472

Pittenger, M. F., Mackay, A. M., Beck, S. C., Jaiswal, R. K., Douglas, R., Mosca, J. D., et al. (1999). Multilineage potential of adult human mesenchymal stem cells. Science 284, 143-147. doi: 10.1126/science.284.5411.143

Ribeiro, C. A., Fraga, J. S., Graos, M., Neves, N. M., Reis, R. L., Gimble, J. M., et al. (2012). The secretome of stem cells isolated from the adipose tissue and Wharton jelly acts differently on central nervous system derived cell populations. Stem Cell Res. Ther. 3, 18. doi: 10.1186/ scrt109

Royo, F., Zuniga-Garcia, P., Sanchez-Mosquera, P., Egia, A., Perez, A., Loizaga, A., et al. (2016). Different EV enrichment methods suitable for clinical settings yield different subpopulations of urinary extracellular vesicles from human samples. J. Extracell. Vesicles 5:29497. doi: 10.3402/jev.v5.29497

Salgado, A. J., Sousa, J. C., Costa, B. M., Pires, A. O., Mateus-Pinheiro, A., Teixeira, F. G., et al. (2015). Mesenchymal stem cells secretome as a modulator of the neurogenic niche: basic insights and therapeutic opportunities. Front. Cell Neurosci. 9:249. doi: 10.3389/fncel.2015.00249

Street, J. M., Barran, P. E., Mackay, C. L., Weidt, S., Balmforth, C., Walsh, T. S., et al. (2012). Identification and proteomic profiling of exosomes in human cerebrospinal fluid. J. Transl. Med. 10, 5. doi: 10.1186/14795876-10-5

Sun, D., Zhuang, X., Xiang, X., Liu, Y., Zhang, S., Liu, C., et al. (2010). A novel nanoparticle drug delivery system: the anti-inflammatory activity of curcumin is enhanced when encapsulated in exosomes. Mol. Ther. 18, 1606-1614. doi: $10.1038 / \mathrm{mt} .2010 .105$

Takeda, Y. S., and Xu, Q. (2015). Neuronal differentiation of human mesenchymal stem cells using exosomes derived from differentiating neuronal cells. PLoS ONE 10:e0135111. doi: 10.1371/journal.pone.0135111

Tan, C. Y., Lai, R. C., Wong, W., Dan, Y. Y., Lim, S. K., and Ho, H. K. (2014). Mesenchymal stem cell-derived exosomes promote hepatic regeneration in drug-induced liver injury models. Stem Cell Res. Ther. 5, 76. doi: $10.1186 /$ scrt465

Tauro, B. J., Greening, D. W., Mathias, R. A., Ji, H., Mathivanan, S., Scott, A. M., et al. (2012). Comparison of ultracentrifugation, density gradient separation, and immunoaffinity capture methods for isolating human colon cancer cell line LIM1863-derived exosomes. Methods 56, 293-304. doi: 10.1016/j.ymeth.2012.01.002

Teixeira, F. G., Carvalho, M. M., Neves-Carvalho, A., Panchalingam, K. M., Behie, L. A., Pinto, L., et al. (2015). Secretome of mesenchymal progenitors from the umbilical cord acts as modulator of neural/glial proliferation and differentiation. Stem Cell Rev. 11, 288-297. doi: 10.1007/s12015-0149576-2

Teixeira, F. G., Carvalho, M. M., Sousa, N., and Salgado, A. J. (2013). Mesenchymal stem cells secretome: a new paradigm for central nervous system regeneration? Cell. Mol. Life Sci. 70, 3871-3882. doi: 10.1007/s00018-013-1290-8

Teng, X., Chen, L., Chen, W., Yang, J., Yang, Z., and Shen, Z. (2015). Mesenchymal stem cell-derived exosomes improve the microenvironment of infarcted myocardium contributing to angiogenesis and anti-inflammation. Cell. Physiol. Biochem. 37, 2415-2424. doi: 10.1159/000438594

Timmers, L., Lim, S. K., Arslan, F., Armstrong, J. S., Hoefer, I. E., Doevendans, P. A., et al. (2007). Reduction of myocardial infarct size by human mesenchymal stem cell conditioned medium. Stem Cell Res. 1, 129-137. doi: 10.1016/j.scr.2008.02.002

Valadi, H., Ekstrom, K., Bossios, A., Sjostrand, M., Lee, J. J., and Lotvall, J. O. (2007). Exosome-mediated transfer of mRNAs and microRNAs is a novel mechanism of genetic exchange between cells. Nat. Cell Biol. 9, 654-659. doi: 10.1038/ ncb1596

van Poll, D., Parekkadan, B., Cho, C. H., Berthiaume, F., Nahmias, Y., Tilles, A. W., et al. (2008). Mesenchymal stem cell-derived molecules directly modulate hepatocellular death and regeneration in vitro and in vivo. Hepatology 47, 1634-1643. doi: 10.1002/hep.22236

Wang, X., Gu, H., Qin, D., Yang, L., Huang, W., Essandoh, K., et al. (2015). Exosomal miR-223 contributes to mesenchymal stem cellelicited cardioprotection in polymicrobial sepsis. Sci. Rep. 5, 13721. doi: 10.1038/srep13721

Willms, E., Johansson, H. J., Mäger, I., Lee, Y., Blomberg, K. E. M., Sadik, M., et al. (2016). Cells release subpopulations of exosomes with distinct molecular and biological properties. Sci. Rep. 6, 22519. doi: 10.1038/srep22519

Wubbolts, R., Leckie, R. S., Veenhuizen, P. T., Schwarzmann, G., Mobius, W., Hoernschemeyer, J., et al. (2003). Proteomic and biochemical analyses of human B cell-derived exosomes. Potential implications for their function and multivesicular body formation. J. Biol. Chem. 278, 10963-10972. doi: 10.1074/jbc.M207550200

Xin, H., Li, Y., Buller, B., Katakowski, M., Zhang, Y., Wang, X., et al. (2012). Exosome-mediated transfer of miR-133b from multipotent mesenchymal stromal cells to neural cells contributes to neurite outgrowth. Stem Cells 30, 1556-1564. doi: 10.1002/stem.1129

Xin, H., Li, Y., Cui, Y., Yang, J. J., Zhang, Z. G., and Chopp, M. (2013a). Systemic administration of exosomes released from mesenchymal stromal cells promote functional recovery and neurovascular plasticity after stroke in rats. J. Cereb. Blood Flow Metab. 33, 1711-1715. doi: 10.1038/jcbfm.2013.152

Xin, H., Li, Y., Liu, Z., Wang, X., Shang, X., Cui, Y., et al. (2013b). MiR$133 \mathrm{~b}$ promotes neural plasticity and functional recovery after treatment of stroke with multipotent mesenchymal stromal cells in rats via transfer of exosome-enriched extracellular particles. Stem Cells 31, 2737-2746. doi: 10.1002/stem.1409

Zarjou, A., Kim, J., Traylor, A. M., Sanders, P. W., Balla, J., Agarwal, A., et al. (2011). Paracrine effects of mesenchymal stem cells in cisplatin-induced renal injury require heme oxygenase-1. Am. J. Physiol. Renal Physiol. 300, F254-F262. doi: 10.1152/ajprenal.00594.2010

Zhang, B., Yin, Y., Lai, R. C., Tan, S. S., Choo, A. B., and Lim, S. K. (2014). Mesenchymal stem cells secrete immunologically active exosomes. Stem Cells Dev. 23, 1233-1244. doi: 10.1089/scd.2013.0479

Zhang, Y., Chopp, M., Liu, X. S., Katakowski, M., Wang, X., Tian, X., et al. (2016a). Exosomes derived from mesenchymal stromal cells promote axonal growth of cortical neurons. Mol. Neurobiol. doi: 10.1007/s12035-016-9851-0 [Epub ahead of print].

Zhang, Y., Chopp, M., Meng, Y., Katakowski, M., Xin, H., Mahmood, A., et al. (2015). Effect of exosomes derived from multipluripotent mesenchymal stromal cells on functional recovery and neurovascular plasticity in rats after traumatic brain injury. J. Neurosurg. 122, 856-867. doi: 10.3171/2014.11.JNS14770

Zhang, Z., Yang, J., Yan, W., Li, Y., Shen, Z., and Asahara, T. (2016b). Pretreatment of cardiac stem cells with exosomes derived from mesenchymal 
stem cells enhances myocardial repair. J. Am. Heart Assoc. 5:e002856. doi: 10.1161/JAHA.115.002856

Zhou, Y., Xu, H., Xu, W., Wang, B., Wu, H., Tao, Y., et al. (2013). Exosomes released by human umbilical cord mesenchymal stem cells protect against cisplatin-induced renal oxidative stress and apoptosis in vivo and in vitro. Stem Cell Res. Ther. 4, 34. doi: 10.1186/scrt194

Zonneveld, M. I., Brisson, A. R., van Herwijnen, M. J., Tan, S., van de Lest, C. H., Redegeld, F. A., et al. (2014). Recovery of extracellular vesicles from human breast milk is influenced by sample collection and vesicle isolation procedures. J. Extracell. Vesicles 3:24215. doi: 10.3402/jev.v3. 24215
Conflict of Interest Statement: The authors declare that the research was conducted in the absence of any commercial or financial relationships that could be construed as a potential conflict of interest.

Copyright $\odot 2016$ Marote, Teixeira, Mendes-Pinheiro and Salgado. This is an openaccess article distributed under the terms of the Creative Commons Attribution License (CC BY). The use, distribution or reproduction in other forums is permitted, provided the original author(s) or licensor are credited and that the original publication in this journal is cited, in accordance with accepted academic practice. No use, distribution or reproduction is permitted which does not comply with these terms. 